\section{The Potential Benefits of PTH Analogs in Prevention of Pathological Fractures in Cerebral Palsy}

\section{Abstract}

Osteoporosis is a detrimental disorder of bone that puts patients at increased risk of pathologic fractures. Patients with cerebral palsy (CP), due to a multitude of factors, are at increased risk of developing this disorder. Studies in the past have looked at various treatment options for these patients to reduce and/or prevent pathologic fractures. Most studies have looked into the use of bisphosphonates for prevention. Teriparatide is a recombinant PTH analog that has been shown to increase bone mineral density and reduce fragility fractures in adults with non$\mathrm{CP}$ related osteoporosis. Despite the reduction seen in these studies, empirical evidence for the use of PTH analogs in CP related osteoporosis is scarce. PTH analogs, like Teriparatide, have shown to be beneficial in patients with non-CP related osteoporosis, further studies need to be performed to assess its benefit for patients with $\mathrm{CP}$ related osteoporosis.

Keywords: Cerebral palsy; PTH analogs; Osteoporosis

\section{Patrisha Shelley, Linda Edwards and Rafik Jaco b*}

Division of Internal Medicine, Department of Medicine, College of Medicine, University of Florida, Jacksonville, Florida, USA

\section{*Corresponding author: Rafik Jacob \\ Đrafik.jacob@jax.ufl.edu}

Assistant Professor, Division of Internal Medicine, Department of Medicine, College of Medicine, University of Florida, Jacksonville, Florida, USA.

Tel: 904-383-1003

Citation: Shelley P, Edwards L, Jacob R (2019) The Potential Benefits of PTH Analogs in Prevention of Pathological Fractures in Cerebral Palsy. J Child Dev Disord. Vol.5 No.3:8

\section{Introduction}

Cerebral palsy (CP) is a group of conditions characterized by central motor dysfunction that leads to impairment in muscle tone, posture and movement. It results from brain injury that occurs during central nervous system development. Life expectancy in this population is shorter than the overall population but has been increasing over the past few decades. Children with CP have a multitude of medical complications due to their disorder. Gastrointestinal reflux, aspiration syndromes, muscle contractures, and seizures are just a few problems associated with CP [1]. These problems follow these patients into their adulthood. In addition to the aforementioned medical complications, CP patients are also at increased risk of developing osteoporosis. Osteoporosis leads to increased morbidity and mortality in this population. In CP patients who suffer from hip fractures, the mortality rate within the first year is $20 \%$ [1]. As CP children progress into adulthood with increasing risk of fractures, interventional trials are needed in this population to determine effective and safe therapeutic agents for the prevention of pathologic fractures [1].

\section{Literature Review}

Osteoporosis is defined as a decrease in bone strength that predisposes individuals to increased risk of fractures [2]. Primary osteoporosis is most often associated with increasing age and alterations in hormonal and nutritional milieu that is detrimental to bone mineral homeostasis. This is most commonly seen in post-menopausal women where estrogen deficiency leads to an imbalance between the activity of osteoclasts and osteoblasts favoring increased bone resorption [2].

The diagnosis of osteoporosis in adults is well defined and is based on assessment of a patient's bone mineral density (BMD) reported as a T-score. A dual energy $x$-ray absorptiometry (DEXA) scan is utilized to measure BMD. A T-score of less than -2.5 is diagnostic for osteoporosis (Figures 1 and 2). In children and young adults, $\mathrm{BMD}$ is reported in a Z-score. The Z-score is adjusted for age and gender. The current definition of osteoporosis in these younger individuals is a BMD Z-score less than -2.0. Certain subsets of individuals, such as CP patients, have a higher risk of developing osteoporosis for a variety of reasons [3].

\section{Osteoporosis in cerebral palsy}

Children and young adults with $\mathrm{CP}$ are at increased risk of osteoporosis and pathologic fractures [4]. Low BMD is a serious problem in patients with $\mathrm{CP}$. One study looking at prevalence of low BMD in the distal femur of CP patients found up to $77 \%$ $(95 \% \mathrm{Cl})$ of patients had z-score lower than -2 [5]. The decrease 


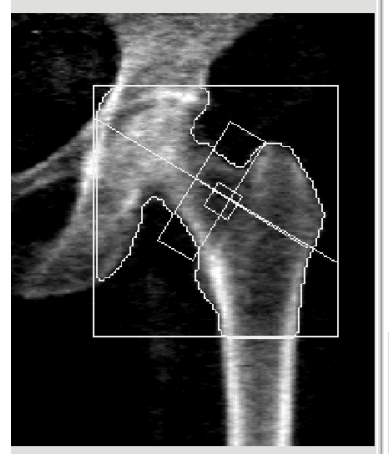

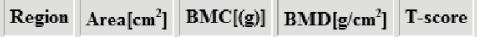
\begin{tabular}{|l|r|r|r|r|}
\hline Neck & 4.30 & 2.16 & 0.504 & -3.1 \\
\hline
\end{tabular}

Figure 1 Osteoporosis in femoral head observed via DEXA scan.

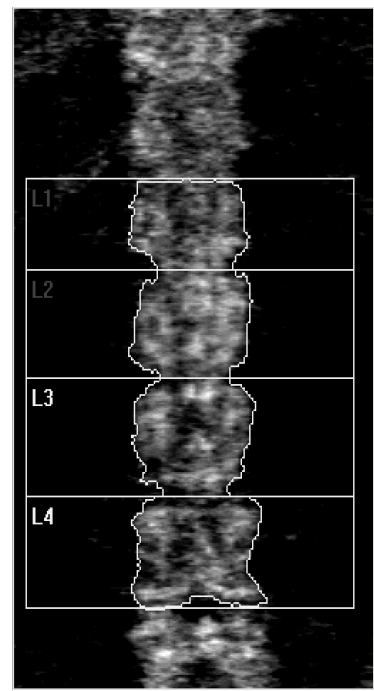

\begin{tabular}{|l|l|l|l|l|}
\hline Region & Area[cm & BMC[(g)] & BMD $\left[\mathrm{g} / \mathrm{cm}^{2}\right]$ & T-score \\
\hline
\end{tabular}

\begin{tabular}{|l|r|r|r|r|}
\hline L3 & 12.79 & 10.48 & 0.820 & -2.4 \\
\hline L4 & 13.60 & 10.61 & 0.780 & -2.6 \\
\hline Total & $\mathbf{2 6 . 3 9}$ & $\mathbf{2 1 . 0 9}$ & $\mathbf{0 . 7 9 9}$ & $-\mathbf{2 . 7}$ \\
\hline
\end{tabular}

Figure 2 Osteoporosis of lumbar spine observed via DEXA scan.

of BMD in this population arises from a multitude of risk factors, including low body mass index (BMI), decreased weight bearing activities, and medications such as anticonvulsant therapies [6]. There is a strong correlation between the severity of immobility and the risk of sustaining fractures. In children with $C P$, the Gross Motor Function Classification System (GMFCS) is used to categorize the functional impairment into five levels (Table 1). Past studies have shown higher incidence of fractures in $C P$ patients in level IV and $\mathrm{V}$ when compared to the more mobile levels I, II and III [6]. Current management consists of weight bearing exercises, increasing time in the sun, vitamin $D$ and calcium supplementation. The addition of bisphosphonates has been recommended in $\mathrm{CP}$ patients who have been diagnosed with osteoporosis and those with previous pathologic fractures. However, the efficacy of this intervention is modest at best as the predominant pathophysiology of osteoporosis in children with CP involves a deficit in the development of bone rather than bone mineral loss commonly observed in postmenopausal women [7-9].

\section{Treatment}

Bone growth is maintained by a process of bone remodeling. This remodeling involves old bone being replaced by new bone. There are five phases involved in bone remodeling: resting, activation, resorption, reversal, and formation. From the resting, or quiescent phase, bone enters activation phase. In the activation phase cells known as osteoclasts are activated and recruited to the bone surface. The resorption phase involves osteoclast resorption of mineral content of the bone. This is followed by the reversal phase, where osteoclasts undergo apoptosis, cell death. Osteoblast cells are then recruited to the surface of the bone. The next phase, formation phase, is when osteoblasts deposit collagen which is mineralized to form new bone [2].

Normal bone growth is dependent on vitamins, minerals, and hormones that play key roles in bone mineral homeostasis. Vitamins $D, C$, and $A$ along with the minerals (calcium, phosphate, and magnesium) are required for normal bone growth. Hormones such as parathyroid hormone (PTH), growth hormone (GH), calcitonin, and sex hormones (estrogen, testosterone) have been implemented in bone mineral homeostasis as well.

The many different treatment options available for osteoporosis work through this remodeling process. Through knowledge of the bone formation the remodeling process can be manipulated to increase bone mineral density.

When there is an imbalance of remodeling activity, a decrease in mineralization, or modifications in hormones there is progression to osteoporosis. Treatment options that focus on the many different factors involved in bone growth have been developed (Table 2) in order to help decrease patient's risk of pathologic fractures that accompany the diagnosis of osteoporosis.

Table 1 Gross Motor Function Classification System [9].

\begin{tabular}{|c|c|}
\hline Level I & Walks without limitations \\
\hline Level II & Walks with limitations \\
\hline Level III & Walks using a hand-held mobility device \\
\hline Level IV & Self-mobility with limitations; may use powered mobility \\
\hline Level V & Transported in a manual wheelchair \\
\hline
\end{tabular}

Table 2 Treatment options for osteoporosis [2].

\begin{tabular}{|c|c|}
\hline Compound & Mechanism of Action \\
\hline $\begin{array}{l}\text { Bisphosphonates: } \\
\text { Alendronate, Risedronate, } \\
\text { Ibandronate, Zoledronic } \\
\text { Acid }\end{array}$ & Decrease osteoclast activity \\
\hline Denosumab & $\begin{array}{l}\text { RANKL Inhibitor; binds to RANK-Ligand on } \\
\text { osteoblast. Leads to a decrease in binding } \\
\text { of RANK receptor on osteoclast, decreasing } \\
\text { osteoclast activation. }\end{array}$ \\
\hline Raloxifene & $\begin{array}{l}\text { Estrogen Agonist/Angtagonist; works } \\
\text { as agonist on bone by decreasing bone } \\
\text { resorption and bone turnover leading to } \\
\text { increased BMD. }\end{array}$ \\
\hline $\begin{array}{c}\text { Teriparatide } \\
\text { Abaloparatide }\end{array}$ & $\begin{array}{l}\text { Parathyroid Hormone Analogues; increasing } \\
\text { osteoblastic activity }\end{array}$ \\
\hline Calcitonin & $\begin{array}{c}\text { Effects of calcitonin on normal human } \\
\text { bone physiology are unclear, receptors } \\
\text { have been discovered on osteoclasts and } \\
\text { osteoblasts }\end{array}$ \\
\hline
\end{tabular}




\section{PTH analogues}

Teriparatide is a parathyroid hormone analogue that works by increasing osteoblast activity. By doing so it leads to increase in bone formation. In 2001, a phase III clinical trial studied the use of teriparatide in postmenopausal women. Over 1600 postmenopausal women were randomized to receive placebo or teriparatide for $\mathbf{2 1}$ months. Compared to the placebo group, teriparatide was shown to increase bone mineral density and reduce vertebral fractures. Fragility fractures at nonvertebral sites such as the hip and wrist were reduced by $53 \%(P<0.05)$ [10]. Teriparatide's use in children is limited due to the increased risk of osteosarcoma due to their open epiphyses [11].

Another medication with similar effects as teriparatide is abaloparatide. Abaloparatide is a parathyroid hormonerelated peptide analog that is approved for the treatment of postmenopausal osteoporosis. In an 18-month long study that compared abaloparatide to placebo found that changes were higher in the abaloparatide group. In the abaloparatide group new vertebral fractures and non-vertebral fractures occurred in $0.6 \%$ and $2.7 \%$, respectively. This is compared to the placebo group with new vertebral fractures at $4.2 \%$ and non-vertebral fractures at $4.7 \% .(p<0.05)$ [12]. Ol is a skeletal dysplasia that is caused by genetic mutations leading to mutations in type 1 collagen. These mutations alter properties of bone matrix and affect osteoblast function. The end result is compromised bone quality putting these patients at increased risk of fractures starting in their childhood and continuing into adulthood. A study in 2014 looked at the use of teriparatide in patients with osteogenesis imperfecta (OI). In this study, 79 adults with OI were randomized to receive teriparatide or placebo for 18 months in a double-blind, placebo-controlled trial. There was an increase in lumbar spine BMD ( $6.1 \%$ increase compared to $\sim 2.8 \%$ increase; $p<0.05)$ and total hip BMD ( $2.6 \%$ increase vs $\sim 2.4 \%$ decrease; $p<0.001$ ) in the teriparatide group. Vertebral BMD increased in the teriparatide group, while a decrease in vertebral BMD was observed in the placebo group ( $18 \%$ increase vs $\sim 5.0 \%$ decrease; $p<0.05$ ) [13].

\section{Bisphosphonates}

Studies using bisphosphonates in $\mathrm{CP}$ patients have shown a

\section{References}

1 Sheridan KJ (2009) Osteoporosis in adults with cerebral palsy. Develop Med Child Neurol 51: 38-51.

2 Tella SH, Gallagher JC (2013) Prevention and treatment of postmenopausal osteoporosis. J Steroid Biochem Mol Biol 142: 155-170.

3 Cranney A, Jamal SA, Tsang JF, Josse RG, Leslie WD (2007) Low bone mineral density and fracture burden in postmenopausal women. Canadian Med Asso J 177: 575-580.

4 Sees JP, Sitoula P, Dabney K, Holmes L, Rogers KJ, et al. (2016) Pamidronate Treatment to Prevent Reoccurring Fractures in Children With Cerebral Palsy. J Ped Orthopaed 36: 193-197.

5 Mergler S, Evenhuis HM, Boot AM, De Man SA, Bindels-De Heus KGCB, et al. (2009) Epidemiology of low bone mineral density and decrease in pathologic fractures and an increase in Z-score. In one study by Henderson et al., at 18 months post treatment there were zero fractures in the group treated with the bisphosphonate pamidronate compared to three fractures in the placebo group. There was an increase in BMD by $89 \%$ (SD 21) in the metaphyseal region of the distal femur compared to $9 \%$ (SD 1 ) in the control group. Z score was seen to improve from -4 (SD 0.6) to -1.8 (SD 1) with pamidronate [1]. Bisphosphonates work by inhibition of osteoclast activity at the cellular level. By decreasing the bone resorption by osteoclast, bisphosphonates lead to an increased amount of bone. This class of drugs is limited by its multitude of adverse effects. With oral therapy, bisphosphonates are well recognized as a cause of esophageal irritation and erosion. Among the more severe complications is osteonecrosis of the jaw $[14,15]$. These complications can limit bisphosphonates use as long-term therapies of osteoporosis. Additionally, long-term studies of bisphosphonates use in CP patients is lacking.

In children with Ol, studies using bisphosphonates showed reduced bone turnover, higher $\mathrm{BMD}$, improved structure and lower fracture rates. In adults with Ol there are fewer studies to evaluate treatment with bisphosphonates. Studies available show an increase in BMD but without a significant decrease in fracture rates [16-19].

\section{Discussion and Conclusion}

As CP patients enter adulthood they are at increased risk of pathologic fractures. These pathologic fractures are associated with increased morbidity and mortality in the general population as well as the CP population. CP patients are a small portion of this population but as they continue to live longer there is an increasing need for effective fracture prevention.

Bisphosphonates have been widely studied in the use of $\mathrm{CP}$ associated osteoporosis. Long term use with bisphosphonates are limited by its multiple adverse events. PTH analogues are a therapy that has been developed and studied in primary osteoporosis but its utility in CP patients is unknown due to lack of studies. Further research into the use of PTH analogues need to be performed to assess the benefit in CP related osteoporosis.

fractures in children with severe cerebral palsy: a systematic review. Develop Med Child Neurol 51: 773-778.

6 Grossberg R, Blackford MG, Kecskemethy HH, Henderson R, Reed MD (2015) Longitudinal assessment of bone growth and development in a facility-based population of young adults with cerebral palsy. Develop Med Child Neurol 57: 1064-1069.

7 Paksu MS, Vurucu S, Karaoglu A, Karacalioglu AO, Polat A, et al. (2011) Osteopenia in children with cerebral palsy can be treated with oral alendronate. Childs Nervous System 28: 283-286.

8 Kim MJ, Kim SN, Lee IS, Chung S, Lee J, et al. (2015) Effects of bisphosphonates to treat osteoporosis in children with cerebral palsy: a meta-analysis. J Ped Endocrinol Metabol 28.

9 Palisano R, Rosenbaum PL, Walter S, Russell D (2008) Gross Motor 
Function Classification System for Cerebral Palsy. Developmental Medicine \& Child Neurology 39: 214-223.

10 Bodenner D, Redman C, Riggs A (2007) Teriparatide in the management of osteoporosis. Clinical Interventions in Aging 2: 499-507.

11 Deal C (2004) The use of intermittent human parathyroid hormone as a treatment for osteoporosis. Curr Rheumatol Rep 6: 49-58.

12 No authors listed (2019) Abaloparatide: Review of a Next-Generation Parathyroid Hormone Agonist. SAGE Journals.

13 Orwoll ES, Shapiro J, Veith S, Wang Y, Lapidus J, et al. (2014) Evaluation of teriparatide treatment in adults with osteogenesis imperfecta. J Clin Invest 124: 491-498.

14 Rodan GA, Fleisch HA (1996) Bisphosphonates: Mechanisms of action. J Clin Invest 97: 2692-2696.
15 Drake MT, Clarke BL, Khosla S (2008) Bisphosphonates: mechanism of action and role in clinical practice. Mayo Clin Proc 83: 1032-1045.

16 Tu KN, Lie JD, Wan CKV, Cameron M, Austel AG, et al. (2018) Osteoporosis: A review of treatment options. PT 43: 92-104.

17 Cranney A, Papaioannou A, Zytaruk N, Hanley D, Adachi J, et al. (2006) Parathyroid hormone for the treatment of osteoporosis: a systematic review. CMAJ 175: 52-59.

18 Harcke HT, Stevenson KL, Kecskemethy HH, Bachrach SJ, Grissom LE (2011) Fracture after bisphosphonate treatment in children with cerebral palsy: The role of stress risers. Ped Radiol 42: 76-81.

19 Fowler EG, Rao S, Nattiv A, Heberer K, Oppenheim WL (2015) Bone density in premenopausal women and men under 50 years of age with cerebral palsy. Arch Phy Med Rehabilitation 96: 1304-1309. 